\title{
TOWARDS CREATIVITY STIMULATING DESIGN INTERVENTION FOR MULTIDISCIPLINARY INNOVATION TEAMS
}

\author{
Välk, Sander; Mougenot, Céline \\ Dyson School of Design Engineering, Imperial College London
}

\begin{abstract}
The aim of our research is to stimulate cross disciplinary design collaboration to improve innovation processes in product and service design domain. We focus on the intersection of biotechnology and design as this field poses great challenges and opportunities for innovation, and it has received little attention in light of technological advancements of digital goods over the past decades. Experimental studies in the area expose challenging interactions, rising from lack of common vocabulary and preconceptions. Organisational management studies suggest that creativity is a prerequisite for innovation in group processes. As such, we are interested in enhancing collective creativity. Numerous studies investigate external creativity triggers, however only on individual level. Our review suggests that external triggers can be effective when the task is problem solving or styling, but ambiguous goals like innovation require stimulation of intrinsic triggers, such as group incidental learning and tacit knowledge. To explain this, we propose a hypothetical innovation approach, that draws attention to cognitive stimulation methods leading to creativity in multidisciplinary teams.
\end{abstract}

Keywords: Multi- / Cross- / Trans-disciplinary processes, Innovation, Creativity

\section{Contact:}

Välk, Sander

Imperial College London

Dyson School of Design Engineering

United Kingdom

sandervaelk@gmail.com

Cite this article: Välk, S., Mougenot, C. (2019) 'Towards Creativity Stimulating Design Intervention for 


\section{INTRODUCTION}

The goal of this literature review is to find novel insight for improving design processes that strategically aim to create innovations in multidisciplinary teams, with particular focus on the intersection between biotechnology and product design. Recently there have been numerous calls from scientific researchers, academics and practicing designers to expand the models of innovation through design thinking, collaboration and scientific discoveries (Gero, 2000; Sawa, 2016; Schindler, 2015; Simons et al., 2011). These calls echo the findings that entangling two or more disciplines (art, design, engineering, science) is the source of innovation, furthermore, new knowledge cannot be produced within one discipline (Oxman, 2016). Similar ideas are shared with scholars from economic sociology and organisational sociology who postulate that innovation only happens across disciplines (Stark, 2009). However, cross disciplinary collaborations propose difficulties in terms of mutual understanding due to "object worlds" and "thought worlds" presented by boundaries between practices (Bucciarelli, 2003). Our research aims to contribute to the understanding about crossing these boundaries.

We investigated six documented studies between biotechnologists and designers (one study consists of several collaborations) to gain understanding about processes, strategies and respective outcomes of their experimental projects. These studies have been chosen due to their experimental nature and lack of predetermined clear problem. Our review revealed a central theme, which can either hinder or enhance the quality of group work: the social nature and characteristics of co-doing. Since we are interested in stimulating innovation within a given context of a project team, and maximising the existing (human) resources, our review also investigates relevant cognitive processes in teams. We have identified that creativity is a key element for innovation in teams (Amabile, 1988). This finding leads us to explore the ways of enhancing creativity. Design researchers have studied the effects of external stimuli on creativity in problem solving exercises and idea creation (Vasconcelos and Crilly, 2016), however we have found that for the purpose of innovation, internal sources of inspiration, motivation and intrinsic triggers can be fruitful aspects to approach (Amabile, 1988), despite lack of research in this domain. However, there is emerging knowledge concerning creativity stimulation, that emphasises ambiguity and un-structured sources of inspiration. These qualities are known to be preferred by designers when they compare and choose creativity triggers in their process (Kwiatkowska et al., 2014).

In this paper, the literature review is arranged as follows: firstly, collaborations between biotechnologists and designers are investigated, secondly the cognitive elements of innovating collaboratively are examined, thirdly different types of creativity triggers and their applicability for innovation are studied. The main outcome of the paper is a approach for creativity and innovation. We also describe a design intervention that will be used to empirically test the proposed approach in the next phase of our research. The findings of the review are expected to be useful for practitioners, researchers and organisations concerned with improving design education, design practice and innovation management.

\section{RELEVANCE OF TEAM CREATIVITY FOR INNOVATION}

Creativity and innovation in all their aspects are vital to the realisation of the potential of human ingenuity and are arguably the essence of design (McMahon et al., 2013). Innovation is particularly essential for organisational survival because of (inter)national competition, changing regulations and rapidly shifting market conditions (Amabile, 1988). The foundation of organisational innovation is built on creative ideas which is directly influenced by individual creativity. However, motivation, expertise and social skills are also required, together with supportive environment offering freedom, good project management, sufficient resources, encouragement and recognition (Amabile, 1988). A study on synergy and team-level creativity describes creativity as the intersection of individual's domain relevant skills, creativity relevant skills and motivation, whereas diversity of the team can aid the creative process by presenting a heterogeneous set of perspectives. Simultaneously, diversity can also hinder group process by limiting common understandings and shared experiences, or by creating a divergence of ideas and styles that result in conflict (Kurtzberg and Amabile, 2001). As multicultural and multidisciplinary teams have become the norm in innovation management and R\&D domains, research is increasingly directed towards understanding challenges and opportunities they pose. This creates new areas of interest for creativity management, particularly when considering design as a social activity, rather than single-practitioner based reflective practice described by Schön (Paletz et al., 2017; Sosa and Gero, 2015). 


\section{COLLABORATION BETWEEN DESIGN AND SCIENCE}

In past, the relationship between science and design was dominated by attempts to make design more scientific and to employ design to make science more understandable. However, it is argued that design can, and should be part of science, but without swamping the unique characteristics of design research with different cultures imported from the arts or sciences (Cross, 2001). Concurrently, instead of bringing science to design, there are calls to bring design to science, because design makes scientific progress visible and usable (Müller, 2017). A concrete strategy for innovation (and increasingly applied in $\mathrm{R} \& \mathrm{D}$ ) is to bring these ideas to life in a form of cross disciplinary collaboration, which is a precondition for novel and useful inventions (Oxman, 2016; Stark, 2009). Additionally, collaborations between designers and biotechnologists can provide the practitioners with novel ways of conceiving the relationships linking science, technology and society (Agapakis, 2014). The challenge is that the interactions between scientists and designers are often complicated due to lack of mutual language, preconceptions about one another and general disinterest (Müller, 2018), however attempts for making similar collaborations more fluent have been made, for example by introducing an engineering-to-biology thesaurus to aid teaching (Nagel et al., 2014).

Enthused by these findings, we study examples from open ended and experimental ventures between biotechnologists and designers as these project examples possess various qualities of environments promoting creativity and innovation: freedom, appropriate resources, good project management, as well as embody important requirements for creative solutions: participants' intrinsic motivation and lack of extrinsic constraints (Amabile, 1988). The sample of collaborations studied is determined by identifying mutual characteristics that are representative throughout - most notably, intrinsic motivation and curiosity combined with pro-active approach from practitioners. Our findings are summarised in Table 1. The general finding is that mutual learning through long term collaboration is a basis for success, in which ever way the studies define success. An additional general finding suggests that open ended approaches and enthusiasm for experimentation are crucial for successful collaboration.

\subsection{Mutual learning and longevity}

An important finding for our review is the importance of longevity of collaborations. This is explicitly illustrated by a Designer in Residence project focussing on algal biotechnology, which found that an intimate insight of being immersed to laboratory activities on a daily basis enables richer experiences than interviews, workshops, short residences or observations when the aim is to design new applications for microscopic algae in the context of urban environment. Long term experimentations have a tendency to eliminate barriers related to language and terminology which naturally occur when different disciplines are combined (Sawa, 2016). Surprisingly, it is noted that ambiguity rising from not knowing the laboratory specific vocabulary in the early stages of the project enhanced mutual learning and facilitated open ended approach, which is an essential aspect of successful implementation of novel discoveries (Amabile, 1988; Sawa, 2016). Furthermore, even when participants in collaborative settings start from widely diverse characteristics, the nature of co-doing will likely induce learning and result in shared understanding on collaborators' work over time (Kurtzberg and Amabile, 2001). These findings are complementary with Schindler's research regarding tacit knowledge transfer and externalising tacit knowledge through experience, nevertheless there are limits to putting merely sensuous knowledge into words which reflects Polanyi's (1983) claim "we know more than we can tell" (Schindler, 2015). Collaboration across different disciplines first requires developing a shared vocabulary and translation of jargon to facilitate communication. For conversations between (synthetic) biology and design fields, misunderstandings can arise not only over specialist language but also over shared terms that have different meanings in different contexts (Agapakis, 2014).

\subsection{Open ended experimentation}

A study in synthetic aesthetics which emerged from synthetic biology (the application of engineering principles to living systems) aimed to bring together biology, art and design in mutually transformative ways by initiating paired exchanges (Calvert and Schyfter, 2017). The authors argue that one of the most valuable skills to learn from the exchange is embracing the experimental and unexpected nature of collaborations. On these grounds, it is suggested that working with artists and designers can enrich social scientific work by helping to develop an emergent form of critique, where the outcomes are not obvious from the outset, but emerge from the process of collaboration itself. In order to reach novel discussions 
and explorations amongst scientists, artists and designers, it is important to be prepared for interactions that are playful, challenging and transcendent.

Operating as a creative practitioner at the intersection of design, biology and technology means to be in a state of determined inventiveness, curiosity and un-knowing (Dunne and Raby, 2013). Such activities assume engagement, interactions without assumptions between spaces and their people, seeking connections and letting go of perceived hierarchies. Such are the reflections from Ginkgo Bioworks Creative Residency program focusing on creating innovative ways of designing with biology (Chieza, 2018). The residency illustrates the importance of building an open environment for multidisciplinary collaboration between synthetic biology and design, by revealing qualities required for this kind of process.

In a review on designing synthetic biology Christina Agapakis (2014) has found that cross disciplinary collaborative experiments and speculative discussions can assist with identifying areas of uncertainty and maintaining a degree of flexibility in response to unanticipated developments. Open-ended strategies and creative methods for design have found their way into the lab through collaborations with designers from many other fields, bringing diverse approaches to the work of biological design. Such collaborations offer engineers the opportunity to imagine new possibilities for how their work might be embedded into the human scale of everyday technology. Through design experiments and speculative prototyping, synthetic biologists can open up new directions of research, propose new questions, and new hypotheses which bridge the biological, technological and social domains. Adapting design methods when developing biological systems allows adaptability, creativity and more importantly, leverages the creation of a vision as demonstrated by collaboration between IDEO (global design firm) and UCSF (synthetic biology lab) (Bernstein, 2011).

Table 1. Review of Design and Science collaborations

\begin{tabular}{|l|c|c|r|}
\hline $\begin{array}{l}\text { Study } \\
\text { reference }\end{array}$ & $\begin{array}{c}\text { (Practitioners') } \\
\text { field of expertise }\end{array}$ & $\begin{array}{c}\text { Goal of the } \\
\text { collaboration or } \\
\text { research }\end{array}$ & Main findings \\
\hline Bernstein, 2011 & $\begin{array}{c}\text { Product design and } \\
\text { synthetic biology }\end{array}$ & $\begin{array}{c}\text { Integrate "design } \\
\text { thinking" into science }\end{array}$ & $\begin{array}{r}\text { Need for a safe space for uncertainty } \\
\text { that is missing in many labs }\end{array}$ \\
\hline $\begin{array}{l}\text { Agapakis, 2014 } \\
\text { Review paper }\end{array}$ & $\begin{array}{c}\text { Synthetic biology } \\
\text { and design }\end{array}$ & $\begin{array}{c}\text { Open up new avenues } \\
\text { for research and design }\end{array}$ & $\begin{array}{r}\text { Design experiments can bridge and } \\
\text { stimulate cross disciplinary domains }\end{array}$ \\
\hline Schinder, 2015 & $\begin{array}{c}\text { Artists, designers, } \\
\text { scientists from } \\
\text { various fields }\end{array}$ & $\begin{array}{c}\text { Artistic research to } \\
\text { develop new musical } \\
\text { instruments }\end{array}$ & $\begin{array}{r}\text { Sensuous knowledge cannot be } \\
\text { verbalised, tacit knowledge transfer } \\
\text { facilitated by shared experience }\end{array}$ \\
\hline Sawa, 2016 & $\begin{array}{c}\text { (Bio) design and } \\
\text { biotechnology }\end{array}$ & $\begin{array}{c}\text { Domestication of algal } \\
\text { biotechnology }\end{array}$ & $\begin{array}{r}\text { Co-invention happens via long term } \\
\text { collaboration and shared vocabulary }\end{array}$ \\
\hline $\begin{array}{l}\text { Calvert and } \\
\text { Schyfter, 2017 }\end{array}$ & $\begin{array}{c}\text { Synthetic biology, } \\
\text { art and design }\end{array}$ & $\begin{array}{c}\text { Speculative and } \\
\text { experimental }\end{array}$ & $\begin{array}{r}\text { Similarities between objectives, } \\
\text { unexpected nature of collaborations }\end{array}$ \\
\hline Chieza, 2018 & $\begin{array}{c}\text { Synthetic biology } \\
\text { and design }\end{array}$ & $\begin{array}{c}\text { Find innovative ways of } \\
\text { designing with biology }\end{array}$ & $\begin{array}{r}\text { Creativity at the intersection of } \\
\text { disciplines requires inventiveness, } \\
\text { and embracing the un-known }\end{array}$ \\
\hline
\end{tabular}

\section{SOURCES OF CREATIVITY FOR INNOVATION}

\subsection{External and internal triggers}

When considering challenges in creativity management, the question of triggering creativity is a natural occurrence. Extensive literature overview on creativity and its associated phenomena such as fixation exists in design research (Crilly and Cardoso, 2017). Majority of design research deals with investigating the effects of external stimuli on designers' creativity in terms of quantity of ideas and quality of ideas during the ideation phase. External triggers, such as showing visual stimuli (images, video, text, animation) to designers when they ideate, can lead to enhanced creativity (Cheng et al., 2014; Sarkar and Chakrabarti, 2008). However, it is crucial to acknowledge that these studies deal with reworking existing artefacts, styling or problem solving. Presenting external stimuli to designers can also result in fixation, which is defined as blind adherence to a set of ideas limiting the output of conceptual design. Design fixation can be seen as cognitive error and therefore 
counterproductive when the aim is to innovate (Crilly and Cardoso, 2017). An empirical study on design fixation investigated the effects of presenting partial photographs to practitioners during ideation process and found that this helped to create more original designs when compared with a group that was presented with full photographs. This positive evaluation can be explained by the fact that successfully dealing with incomplete information contributes to the sense of achievement, however it is acknowledged that this technique is not applicable for new product development and innovation (Cheng et al., 2014). As such, we assume that innovation processes require and rely on intrinsic sources of creativity on a more significant scale than reflected in existing research and suggest that ambiguity can serve as a creativity trigger.

\subsection{Complexity of stimulating internal triggers}

Emotion driven design processes are a gradually growing stream of design research that focuses on understanding the emotions of product's users, and on the development of tools and techniques that facilitate an emotion-focused design process (Desmet and Stappers, 2011). There is a direct link between emotion driven processes and their outcome with designer's own emotional arousal. Therefore, it can be impactful to stimulate designers' own emotions in order to enhance creativity (Mougenot et al., 2012). These findings suggest that when the aim is to innovate and trigger creativity, the stimulation process is far more multifaceted and complex than mere reliance on an external stimulus. Intervening socio-affective dimension of group dynamics is known to have an effect on group creativity. For example there are studies that show how time pressure can enhance productivity and support collaborative learning (Mougenot et al., 2017). Another study on team creativity has identified the importance of trust in team work, which is based on emotional bonds and perceived competencies of individual members (Barczak, 2010). Emotions are known to grow out of social interactions, thus emotion is a pervasive influence in teams and fundamental for determining interaction characteristics between team members. Emotionally intelligent teams create both cognitive and affective team trust which is a desirable characteristic for innovation.

Methods for providing procedural influences on creativity and innovation have also been identified. It appears that creativity can be encouraged within work groups through autonomy, encouragement of creativity, mutual openness to ideas, constructive challenge to new ideas, and shared goals and commitments (Kurtzberg and Amabile, 2001). It is clear, that these factors rise from the social context of work, while impacting the individual's performance. As such, focussing on group cognition and composition could be an insightful aspect to study when dealing with creativity management. Studies in technological R\&D multidisciplinary design teams have focussed on the reframing (the process to find a new productive frame for subsequent activities) aspect in collaborative design teams and found that surprises have an impact on group process. More specifically, surprises incite reframing and are a source of team learning leading to innovation (Stompff et al., 2016).Studies have found that addressing cognitive and knowledge-based approaches to triggering creativity can be effective and meaningful when dealing with issues regarding innovation. Rietzschel et al. (2007) have found that creativity can be enhanced by inducing people to leave the path of least resistance and argue for the importance of domain knowledge in activities aiming for quantity and quality of ideas.

We have described a list of factors that are likely to have an effect on creativity, ranging from stimulating emotions to increasing knowledge base of the practitioner. We propose an umbrella term internal sources of creativity, in order to refer to these complex phenomena. These factors are seen as contrasting to external stimuli as inspiration sources described in design research (Vasconcelos and Crilly, 2016). In the next chapter of our research we are aiming to gain a better understanding on cognition and complex factors that investigate cognition in groups. Majority of design research focuses on creativity as a single practitioner-based phenomenon, however little research has been done to investigate the process of group creativity (Amabile et al., 1996; Crilly and Cardoso, 2017).

\section{COGNITIVE PROCESSING IN GROUP CREATIVITY}

\subsection{Learning by doing}

There is little empirical research in the areas of new product development and design innovation that investigates the social dimensions of innovation (Smulders and Brehmer, 2012). A study on reframing 
in multidisciplinary design team explores the social dimension of collaborative design from the perspective on Schön's reflective practice and identifies two phases in the design process: sensemaking and future framing. It finds that unexpected events during early stages of the design process, where uncertainty and ambiguity prevail, there are benefits to exploring and discovering new frames for finding novel opportunities as this is the source for team learning and innovation (Stompff et al., 2016). Team learning is a phenomenon related to situated cognition. The theory of situated cognition rejects the assumption that learning is the reception of factual knowledge or information. Instead, it is put forward that learning is a process of participation in communities of practice, participation that is at first legitimately peripheral but that increases gradually in engagement and complexity (Lave and Wenger, 1991). When aiming to develop innovations, the task is essentially complex, therefore we assume that it requires an element of mutual learning and co-doing as a group, before truly novel and appropriate ideas can be achieved. It is important to stress that this type of learning can be intentional, but is never highly structured, which makes it incidental (Marsick and Watkins, 2001). Informal or also known as incidental learning is always occurring, with or without the practitioner being aware of it. As learning grows out of everyday encounters while working and living in a given context, it poses an important perspective for creativity management and strategy. Marsick and Watkins characterise incidental learning as being integrated with daily routines, triggered by internal or external jolt, not highly conscious, influenced by chance, inductive of reflection and action, linked to learning of others. The process of collaboration between biotechnologists and designers in reviewed papers can be viewed through the lens of incidental situatedness. This perspective provides an insight into the details of co-doing and learning by doing, which are important for developing strategies that aim to enhance creativity in multidisciplinary innovation collaborations.

\subsection{Creating and sharing new knowledge}

In the biotechnology and science collaborations reviewed earlier, creating new knowledge was a key element for fruitful cross-disciplinary collaboration. This is reflected in mutual understanding about the benefits and potentialities of such ventures by parties involved from different domains. Such unanimous understanding suggests that practitioners were able to form a mutual understanding, which originates in sharing and synthesising knowledge.

In an economy where the only certainty is uncertainty, the one sure source of lasting competitive advantage is knowledge. Herbert Simon (1997) views an organisation as a machine for "information processing", however numerous successful Japanese companies (such as Sharp, Honda, Canon) have developed a proactive way to approach knowledge creation and its role in the organisation. It is argued that the success of these organisations relies on managing the creation of new knowledge by tapping individual insights and making these insights available collectively. Part of managing this knowledge is in converting tacit knowledge to implicit (Nonaka and Takeuchi, 1995). There have been numerous studies investigating ways of sharing tacit knowledge (defined as knowledge we use unconsciously and cannot entirely put into words) for example by using multimedia to transfer craft skills from experienced to novice practitioners (Schindler, 2015; Wood, 2005). Similarly, in design research there have been attempts to leverage tacit knowledge by using prototypes in the context of automotive product development (Pedersen, 2016). Furthermore, studies on internal, reflective prototyping in an industry setting have proven to leverage tacit knowledge transferral (Erichsen et al., 2016). Nonaka and Takeuchi have suggested that a way to make tacit knowledge available is by means of a metaphor. Metaphors allow to construct knowledge in new ways and begin to express knowledge what is known in new ways as well as allow expression of things one cannot yet say. As such, metaphors are highly effective in fostering direct commitment to the creative process in the early stages of knowledge creation (Nonaka and Takeuchi, 1995). These ideas have also found successful implementation in innovation driven design approaches that aim to produce interaction centred user experiences (Hekkert and van Dijk, 2014). These researchers have acknowledged and demonstrated how metaphors trigger knowledge creation process and together with analogies formulate new knowledge. This allows for a more equivocal vision, which gives practitioners in multidisciplinary collaborative teams the freedom and autonomy to set their own goals. A study in cognitive ethnography postulates that cognition is always situated in complex sociocultural world and affected by it. Adaptability of cognition is influenced by given surroundings, and therefore should always be studied in social and cultural "real world" or so called natural habitat (Hutchins, 1995). Understanding the knowledge-based nature of the biotechnology and 
design collaborations can leverage the creation of suitable strategies and hypothetical design interventions that aim to enhance creative quality in ideas generated.

\section{TOWARDS AN APPROACH FOR CREATIVITY, BASED ON DESIGN \& SCIENCE COLLABORATION}

\subsection{Approach for future collaborations}

According to Schön, a key characteristic of design is the search for messy and problematic solutions. Simon's view on 'science of design' incorporates solving problems that are well-formed in advance (Cross, 2001). Contrastingly, Rittel \& Webber propose that the classical science and engineering paradigm is not applicable for problems of open societal systems and planning problems are inherently wicked. Design as a discipline of planning therefore poses a great deal of complexity and ambiguity, especially so, when the aim is to create something completely novel and useful. There are examples in design research that address this ambiguous nature of design by deliberately producing artefacts that facilitate open ended approach and interactions in behavioural design to achieve desired effects on people (Boon et al., 2018). This suggests that embracing ambiguity instead of trying to control or prevent it in innovation processes can have positive outcomes.

We suggest that traditional ways of stimulating creativity by introducing external stimuli (Cheng et al., 2014; Vasconcelos and Crilly, 2016) are not necessarily most fruitful when the aim is to solve wicked problems or to innovate. Instead, stimulating internal incidental triggers, as suggested in Figure 1, can lead to more meaningful and innovative outcomes. We investigated empirical studies at the intersection of design and biotechnology, including synthetic biology, together with theoretical studies about group cognition and creativity. The key characteristics enhancing performance in these projects have been identified as mutual learning, open ended approach and longevity of collaborations. In context of examined experiments, we observed a lack of clear strategy or explicit incentive for a predefined end result.

Based on the aforementioned studies, we hypothesise that, for cross disciplinary collaborations (e.g. design and biotechnology), having a broad goal such as social innovation can be an appropriate aim, and there could be benefits about having a strategic process or approach for such ventures.

As creativity can be triggered through external stimuli for problem solving purposes, we suggest that in similar manner, creativity can be effectively enhanced through strategical stimulation of internal triggers related to team cognition, if the aim is to innovate or tackle a wicked problem. Consequently, we propose an approach for enhancing creative quality of ideas generated in design and science collaborations, when the aim is to innovate (Figure 1).

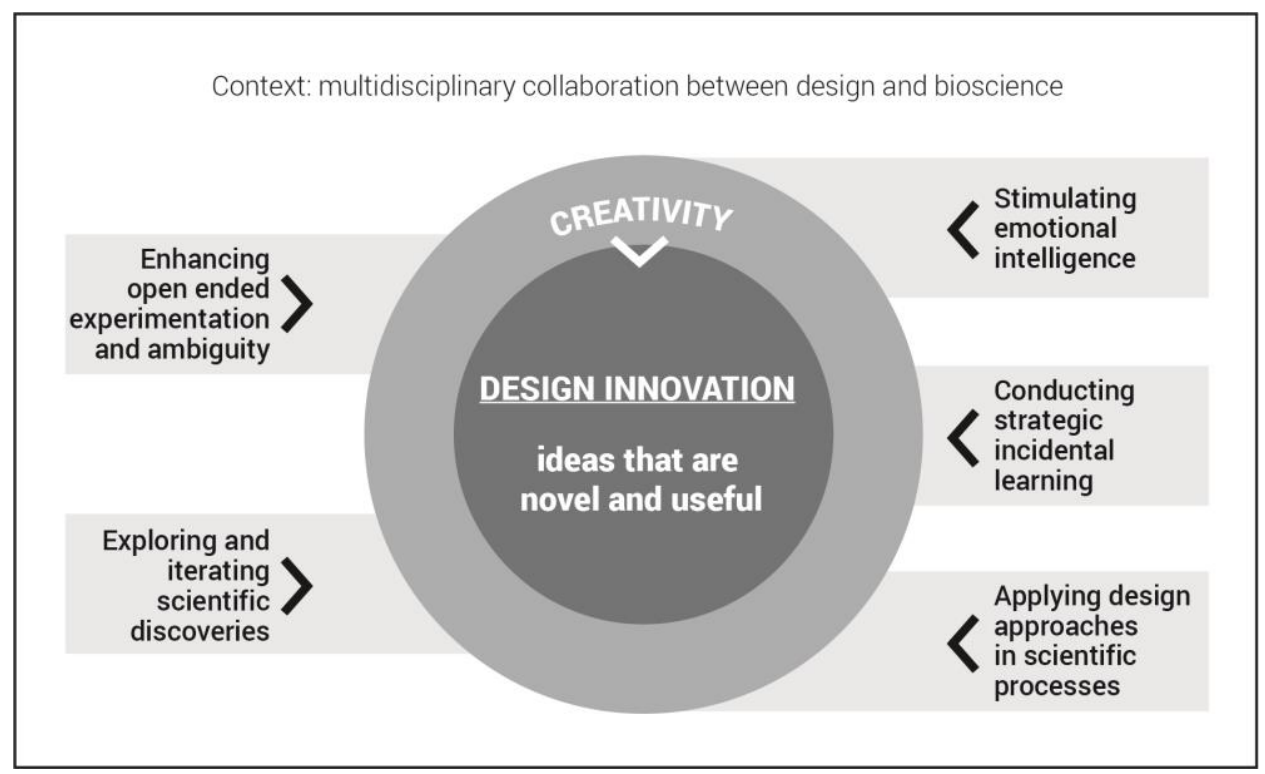

Figure 1. Hypothetical approach for stimulating creativity through five key activities 


\subsection{Towards a design intervention}

In order for internal triggers of practitioners to become driving force in collaborations, it should be ensured that the approach or intervention doesn't hinder the open-ended nature required for creativity and innovation as found in organisational creativity and innovation studies as well as in design innovation management research. Given the social nature of innovation within organisations, we have also explored the cognitive processes in teams and found that characteristics such as openness and transparency combined with motivation, expertise and creativity support the creation of innovative ideas. Emotional intelligence in teams leads to cognitive and affective trust, which is also a supporting factor for creativity.

Our study opens up a set of research directions which have the potential to enhance creativity and innovation through addressing questions related, but not limited to:

- Design for emotional intelligence in design and biotechnology collaborations

- How to use scientific discoveries as sources of inspiration for design?

- How to collaboratively apply design approaches in the development of biotechnological innovations?

- How to use incidental learning as a means to unlock creativity and innovation?

- How to strategically conduct incidental learning to improve team creativity?

- How to use ambiguity as a means to unlock the creativity of multidisciplinary groups?

- How to accelerate science and design collaborations?

For the next phase of our research, the aim is to conduct a series of empirical experiments which see a design intervention being introduced to the context of collaboration between designer(s) and biotechnologist(s). The aim of this design intervention will be to stimulate creativity and build a bridge to innovations. Cognitive processes regarding motivation and creativity suggest that the intervention should be subtle and facilitate bottom up approach, while bearing in mind ethical considerations. Other implications from our findings, on the next stage of the research to be considered are the ability to facilitate cross disciplinary knowledge creation and sharing, transfer of tacit knowledge to explicit knowledge, encouragement of experimentation and open-ended approach, freedom and ability to interpret tasks to formulate meaningful goals, and emphasis on intrinsic motivators.

We have set out to run collaborative idea generation sessions on a predefined broad topic with synthetic biologists and designers. In the first stage, the aim is to conduct an ethnographic study with a control group with focus on co-evolution of ideas in conversations by applying a protocol suggested for analysing generative conversations by Reymen, Dorst and Snelders (McDonnell and Lloyd, 2009). Particular focus in our experiment will be on identifying boundary objects (Carlile, 2002; Star and Griesemer, 1989) and reframing (Schön, 1991). These phenomena suggest that learning in conversations has occurred. The eventual goal is to understand the correlation between this learning and creative quality of ideas generated in collaborations (Cross et al., 1992). Once an understanding about (incidental and situated) learning in these collaborations has emerged, we will use that knowledge to design an intervention with the aim to trigger or stimulate creativity. Consequently, similar collaborative idea generation sessions will be run with the intervention introduced to the context. We will then analyse the impact of the intervention on the creative quality of ideas and compare to control group.

The expected outcome of the research is a new model of innovation, supported by a toolkit that can be implemented in practice as well as academia.

\section{REFERENCES}

Agapakis, C.M. (2014), "Designing synthetic biology”, ACS Synthetic Biology, Vol. 3 No. 3, pp. 121-128. Amabile, T.M. (1988), "A Model of Creativity and Innovation in Organizations", Research in Organizational Behaviour, Vol. 10 No. 1, pp. 123-167.

Amabile, T.M., Conti, R., Coon, H., Lazenby, J. and Herron, M. (1996), “Assessing the Work Environment for Creativity Assessing The Work Environment For Creativity University of Michigan University of Southern California", Management, Vol. 39 No. 5, pp. 1154-1184.

Barczak. (2010), "Antecedents of Team Creativity: An Examination of Team Emotional Intelligence, Team Trust and Collaborative Culture", Creativity and Innovation Management, Vol. 19 No. 1, pp. 332-345.

Bernstein, R. (2011), "Drop that pipette: Science by design”, Cell, Elsevier Inc., Vol. 147 No. 3, pp. 496-497. 
Boon, B., Rozendaal, M.C. and Stappers, P.J. (2018), “Ambiguity and Open-endedness in Behavioural Design”, Proceedings of the DRS 2018 International Conference: Catalyst, pp. 2075-2085.

Bucciarelli, L.L. (2003), "Designing and learning: A disjunction in contexts”, Design Studies, Vol. 24 No. 3, pp. 295-311.

Calvert, J. and Schyfter, P. (2017), "What can science and technology studies learn from art and design? Reflections on 'Synthetic Aesthetics"”, Social Studies of Science, Vol. 47 No. 2, pp. 195-215.

Carlile, P.R. (2002), “A Pragmatic View of Knowledge and Boundaries: Boundary Objects in New Product Development”, Organization Science, Vol. 13 No. 4, pp. 442-455.

Cheng, P., Mugge, R. and Schoormans, J.P.L. (2014), “A new strategy to reduce design fixation: Presenting partial photographs to designers”, Design Studies, Elsevier Ltd, Vol. 35 No. 4, pp. 374-391.

Chieza, N. (2018), "Reflections from Ginkgo's first creative-in-residence”, Gingko Bioworks Blog.

Corvin, T., Pennington, M., Veyrier, C.-A., Huron, S., Arai, K., Baker, M., Mougenot, C. et al. (2017), "Tensions in Creativity Workshops", Proceedings of the European Conference on Cognitive Ergonomics 2017 - ECCE 2017, pp. 93-100.

Crilly, N. and Cardoso, C. (2017), "Where next for research on fixation, inspiration and creativity in design?", Design Studies, Elsevier Ltd, Vol. 50, pp. 1-38.

Cross, N. (2001), "Designerly Ways of Knowing: Design Discipline Versus Design Science”, Design Issues, Vol. 17 No. 3, pp. 49-55.

Cross, N., Dorst, K. and Roozenburg, N. (1992), "Research in Design Thinking”, Delft University Press, pp. 111-118.

Desmet, P.M.A. and Stappers, P.J. (2011), “Editorial”, CoDesign, Vol. 7 No. 2, pp. 61-64.

Dunne, A. and Raby, F. (2013), "Speculative Everything", Design, Fiction and Social Dreaming.

Erichsen, J.A.B., Pedersen, A.L., Steinert, M. and Welo, T. (2016), "Learning in Product Development: Proposed Industry Experiment Using Reflective Prototyping”, Procedia CIRP, The Author(s), Vol. 50 No. 3, pp. 454-459.

Gero, J.S. (2000), “Computational Models of Innovative and Creative Design Processes”, Technological Forecasting and Social Change, Vol. 64 No. 2-3, pp. 183-196.

Hekkert, P. and van Dijk, M. (2014), Vision in Design, 1st ed., BIS, available at: https://www.bispublishers.com/vision-in-product-design.html.

Hutchins, E. (1995), Cognition in the Wild, MIT Press, available at: https://doi.org/10.1023/A:1008642111457.

Kurtzberg, T.R. and Amabile, T.M. (2001), "From Guilford to Creative Synergy: Opening the Black Box of Team-Level Creativity”, Creativity Research Journal, available at: https://doi.org/10.1207/S15326934CRJ1334_06.

Kwiatkowska, J., Szóstek, A. and Lamas, D. (2014), “(Un) structured Sources of Inspiration : Comparing the Effects of Game-like Cards and Design Cards on Creativity in Co-design Process”, Pdc '14, No. 2010, pp. 31-39.

Lave, J. and Wenger, E. (1991), Situated Learning: Legitimate Peripheral Participation (Learning in Doing: Social, Cognitive and Computational Perspectives), Learning in Doing, available at: https://doi.org/10.2307/2804509.

Marsick, V.J. and Watkins, K.E. (2001), "Informal and Incidental Learning”, New Directions for Adult and Continuing Education, Vol. 2001 No. 89, p. 25.

McDonnell, J. and Lloyd, P. (2009), About Designing:Analysing Design Meetings, 1st ed., CRC Press, Balkema, pp. 67-82.

McMahon, C., Lindemann, U., Gero, J.S., Leifer, L., Steinert, M., Edmonds, E., Goldschmidt, G. et al. (2013), "Perspectives on design creativity and innovation research", International Journal of Design Creativity and Innovation, Vol. 1 No. 1, pp. 1-42.

Polanyi, M. (1983), The Tacit Dimension, Peter Smith, Gloucester, MA available at: https://doi.org/http://www.worldcat.org/title/tacit-dimension/oclc/10401108.

Mougenot, C., Ikeda, H. and Watanabe, K. (2012), "Influence of Designers 'Kansei on the Design Outcomes", Keer, Vol. 12 No. 2, pp. 245-250.

Müller, B. (2017), "Bringing Design to Science - Science can benefit more from design than design from science", Www.Medium.Com, available at: https://medium.com/@borism/bringing-design-to-science3fa653f2c149 (accessed 6 December 2018).

Müller, B. (2018), "Strategies for Design-Science Collaborations”, Www.Medium.Com, available at: https://medium.com/@borism/strategies-for-design-science-collaborations-10199f3b8305 (accessed 1 December 2018).

Nagel, J.K.S., Nagel, R.L. and Eggermont, M. (2014), “Teaching Biomimicry With an Engineering-to-Biology Thesaurus", No. May 2015, p. V001T04A017.

Nonaka, I. and Takeuchi, H. (1995), "Knowledge-Creating Company", Harvard Buisiness Review, available at: https://doi.org/10.1016/S0969-4765(04)00066-9.

Oxman, N. (2016), “Age of Entanglement”, Design and Science, MIT Media Lab, No. MIT Press, pp. 1-11. 
Paletz, S.B.F., Sumer, A. and Miron-Spektor, E. (2017), "Psychological factors surrounding disagreement in multicultural design team meetings", Analysing Design Thinking: Studies of Cross-Cultural Co-Creation, Taylor \& Francis, Vol. 0882, pp. 41-58.

Pedersen, A.L. (2016), “Using Prototypes to Leverage Tacit Knowledge Jørgen Andreas Bogen”, No. June.

Rietzschel, E.F., Nijstad, B.A. and Stroebe, W. (2007), "Relative accessibility of domain knowledge and creativity: The effects of knowledge activation on the quantity and originality of generated ideas", Journal of Experimental Social Psychology, Vol. 43 No. 6, pp. 933-946.

Rittel, H. and Webber, M. (1973), "Dilemmas in a General Theory of Planning”, Policy Sciences, Vol. 4 No. December 1969, pp. 155-169.

Sarkar, P. and Chakrabarti, A. (2008), "The effect of representation of triggers on design outcomes", Artificial Intelligence for Engineering Design, Analysis and Manufacturing: AIEDAM, Vol. 22 No. 2, pp. 101-116.

Sawa, M. (2016), "The laboratory life of a designer at the intersection with algal biotechnology", Architectural Research Quarterly, Vol. 20 No. 1, pp. 65-72.

Schindler, J. (2015), "Expertise and tacit knowledge in artistic and design processes: Results of an ethnographic study", Journal of Research Practice, Vol. 11 No. 2, pp. 1-23.

Schön, D. (1991), The Reflective Practitioner - How Professionals Think in Action, Routledge of Taylor \& Francis Group, New York, available at: https://www.taylorfrancis.com/books/9781351883160.

Simon, H.A. (1997), The Sciences of the Artificial (3rd Ed.), Computers \& Mathematics with Applications, available at: https://doi.org/10.1016/S0898-1221(97)82941-0.

Simons, T., Gupta, A. and Buchanan, M. (2011), "Innovation in R \& D: Using design thinking to develop new models of inventiveness, productivity and collaboration", Journal of Commercial Biotechnology, Vol. 17 No. 4, pp. 301-307.

Smulders, F.E.H.M. and Brehmer, M. (2012), "Innovating innovation: Towards a NPD-management taxonomy", Proceedings from the 13th International CINet Conference, Rome, Italy, 16-18 September 2012, pp. 9871002.

Sosa, R. and Gero, J.S. (2015), "EDAM : A computational study of creativity in design : The role of society A computational study of creativity in design: The role of society", No. November 2005, pp. 229-244.

Star, S.L. and Griesemer, J.R. (1989), "Institutional Ecology, Translations and Boundary Objects: Amateurs and Professionals in Berkeley’s Museum of Vertebrate Zoology, 1907-39”, Social Studies of Science, Vol. 19 No. 3, pp. 387-420.

Stark, D. (2009), The Sense of Dissonance, Princeton University Press, available at: https://doi.org/10.1515/9781400831005.

Stompff, G., Smulders, F. and Henze, L. (2016), "Surprises are the benefits: reframing in multidisciplinary design teams", Design Studies, Elsevier Ltd, Vol. 47, pp. 187-214.

Vasconcelos, L.A. and Crilly, N. (2016), "Inspiration and fixation: Questions, methods, findings, and challenges", Design Studies, Elsevier Ltd, Vol. 42, pp. 1-32.

Wood, N. (2005), "Unknown knowns; uncovering tacit knowledge for the design of interactive media", No. May, pp. 26-29. 